\title{
Dynamic Oromandibular Reconstructions Using Chimeric Fibular and Gracilis or Vastus Lateralis Free Flaps
}

\author{
Min Ji Kim, MD, $\mathrm{PhD}^{1}$ Jong Woo Choi, $\mathrm{MD}, \mathrm{PhD}^{2}$ \\ Seung Ho Choi, MD, PhD ${ }^{3}$ \\ ${ }^{1}$ Department of Plastic and Reconstructive Surgery, Ajou Medical Center, \\ College of Medicine, Ajou University, Suwon, Republic of Korea \\ 2 Department of Plastic and Reconstructive Surgery, College of \\ Medicine, Seoul Asan Medical Center, University of Ulsan, Seoul, \\ Republic of Korea \\ ${ }^{3}$ Department of Otolaryngology, College of Medicine, Seoul Asan \\ Medical Center, Ulsan University, Seoul, Republic of Korea
}

Woo Shik Jeong, MD ${ }^{2}$ Soon Yuhl Nam, MD, $\mathrm{PhD}^{3}$

Address for correspondence Jong Woo Choi, MD, PhD, MMM, Department of Plastic and Reconstructive Surgery, College of Medicine, Asan Medical Center, University of Ulsan, 88 Olympic-ro 43gil, Songpa-gu, Seoul 05505, Republic of Korea (e-mail: pschoi@amc.seoul.kr).

J Reconstr Microsurg Open 2020;5:e36-e42.

\begin{abstract}
\section{Keywords}

- oromandibular defect

- fibular free flap

- chimeric flap

- functional head and neck reconstruction

- dynamic reconstruction

Background Despite efforts of head and neck functional reconstruction, anatomic restoration has been used until now. This article describes our experience of using a chimeric free fibular osteocutaneous flap conjoined with a functional muscle free flap, defined as dynamic oromandibular reconstruction.

Methods Through a retrospective chart review, four consecutive patients who underwent reconstruction with a total fibular free flap were included. The former two patients underwent reconstruction using a conventional osteocutaneous free fibular flap. The latter two patients had an oromandibular defect after cancer resection and underwent dynamic oromandibular reconstruction with a chimeric free fibular flap conjoined with a gracilis free flap or vastus lateralis muscle free flap.

Results In the speech analysis, the dynamic group revealed a satisfactory tongue movement. Protrusion and lateralization were the most different movement changes. The tongue range of motion score was 62.5 in the dynamic group and 25.0 in the control group. On the dynamic magnetic resonance imaging, the contact of the soft palate with the tongue was excellent, and the epiglottis closure during deglutition was complete. In the three-dimensional volumetric analysis of mandibular aesthetic contouring, the dynamic group showed a much smaller difference in hemifacial volume, with a difference of $73.7 \mathrm{~mL}$ in the dynamic group and $101.76 \mathrm{~mL}$ in the control group.

Conclusion This study is a preliminary trial of dynamic oromandibular reconstruction using chimeric free fibular flaps with functional muscle transfer. We demonstrated the possibility of dynamic oromandibular reconstruction, which enhanced more functional aspects in the patients in this study.
\end{abstract}

An extensive oromandibular defect after oral cavity cancer resection is one of the most challenging aspects of head and neck reconstruction. The current advances in microsurgical free flaps have enabled the use of double free flaps for covering extensive defects. The concept is that each defect can be matched with each selected flap; thus, some surgeons use dual free flaps as their preferred method. ${ }^{1,2}$

received

December 11, 2019

accepted

May 17, 2020
DOI https://doi.org/

10.1055/s-0040-1713801. ISSN $2377-0813$
In spite of the relatively successful outcomes, we think that this concept aims at anatomical restoration. Given the fact that the tongue is one of the most dynamic structures in our body, simple coverage of the tongue and mandible defects cannot guarantee the patient's postoperative quality of life. We realize that we have been missing an important part of functional impairment when treating intraoperative defects. The defect

Copyright $\odot 2020$ by Thieme Medical Publishers, Inc., 333 Seventh Avenue, New York, NY 10001, USA. Tel: +1(212) 760-0888.
License terms

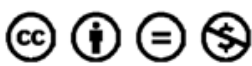


involves a mandibular bony defect and soft tissues such as the oral mucosa or tongue/base of the tongue, and commonly involves various submental muscles related to tongue function.

Feeding and speech are the most concerning functions in head and neck reconstruction. In our experience, tongue movement is commonly restricted after mandible reconstruction with a fibular free flap in which the submental muscles related to the tongue movement are resected. Tongue movement is controlled by the extrinsic/intrinsic muscles of the tongue. ${ }^{3}$ In the jaw-hyoid-tongue complex, hyoid movement is linked to the masticatory/chewing cycle and their relative relationship affects the range of motion (ROM) of the oral floor. This hyolingual apparatus also affects the oropharyngeal surface of the tongue. ${ }^{4-6}$ Patients with oromandibular cancer show impairment of this tongue-jaw interaction. Thus, a dynamic functional approach through comprehensive reconstruction is required to improve the quality of life in such cases.

We hypothesized that dynamic oromandibular reconstruction is possible on the basis of the concept of using a chimeric flap with a free fibular osteocutaneous flap conjoined with a functional muscular flap such as the gracilis muscle free flap. All the extrinsic and intrinsic tongue muscles except one muscle are innervated by the hypoglossal nerve. While the osteocutaneous flap can provide a rigid mandibular reconstruction, the conjoined gracilis or vastus lateralis muscular flap can replace the genioglossus muscle function. Finally, the chimerization and reinnervation give rise to a dynamic system.

This article describes our experience with oromandibular reconstruction using a chimeric free fibular osteocutaneous flap conjoined with a functional muscle free flap. To achieve a dynamic reconstruction, the method of designing the chimeric flap and its functional comparison with the conventional fibular free flap are detailed herein.

\section{Methods}

For adequate comparison of the surgical details, we included four consecutive patients. The former two patients underwent reconstruction with a conventional osteocutaneous free fibular flap. The latter two patients had an oromandibular defect after cancer resection and underwent a dynamic oromandibular reconstruction with a chimeric free fibular flap conjoined with a gracilis free flap (-Table $\mathbf{1}$ ).

\section{Fibula Osteocutaneous Free Flap}

Preoperatively, the mandibular resection margin and type of reconstruction were planned. Thus, we manufactured a three-dimensional (3-D) printing cutting guide (Jeil Medical, Seoul, Republic of Korea) based on 3-D simulation (-Fig. 1). With a cutting guide, the proximal osteotomy site can be estimated. The patient was positioned supine with knee flexion. With a handheld Doppler device, after marking with a skin perforator, dissection was performed via the posterior approach. ${ }^{7}$ The distal soleus and flexor hallucis longus muscles are detached from the fibula, and all the branches and perforators are traced until the peroneal vessels were reached. After completing the posterior dissection, the anterior skin paddle was then incised. A 1- to 2-mm muscle cuff was left at the lateral border of the fibula. In this study, distal osteotomy was performed first, followed by proximal osteotomy. The dissection of the interosseous membrane enabled mobilization of the fibular segments. For the chimerization of other flaps, the distal pedicle length should remain $>2 \mathrm{~cm}$ longer than the needed length. After completion of the osteotomy and interosseous membrane cutting on both sides, the pedicle could be freely skeletonized. Cutting the proximal pedicle should be delayed until completion of the chimerization with a dynamic muscular flap through microanastomosis (-Fig. 2). For the donor site, split-thickness skin grafts were placed after muscle repair.

\section{Case 1: Chimeric Gracilis Muscle Flap}

A 51-year-old woman with an adenoid cystic carcinoma in the oral floor underwent resection of the oral floor along with the base of the tongue and a bilaterally modified radical neck

Table 1 Patient demographic and baseline characteristics

\begin{tabular}{|l|l|l|l|l|}
\hline Patient no. & $\mathbf{1}$ & $\mathbf{2}$ & $\mathbf{3}$ & $\mathbf{4}$ \\
\hline Type of reconstruction & Dynamic & Dynamic & Control & Control \\
\hline Age (y) & 51 & 52 & 69 & 56 \\
\hline Sex & F & F & F & M \\
\hline Comorbidity & $(-)$ & $(-)$ & DM, HTN & $(-)$ \\
\hline Pathology & Mouth floor cancer & Gingival cancer & Mouth floor cancer & Gingival cancer \\
\hline Stage & cT4aN0M0 & cT4aN2cM0 & cT4aN1M0 & cT4N0M0 \\
\hline Neck dissection & Bilateral SND & Bilateral SND & Ipsilateral SND & Ipsilateral SND \\
\hline Chemotherapy & $(-)$ & $(-)$ & $(-)$ & $(-)$ \\
\hline Radiotherapy & $(+)$ & $(-)$ & $(+)$ & $(+)$ \\
\hline Length of hospital stay (d) & 27 & 51 & 21 & 19 \\
\hline Follow-up (mo) & 14 & 10 & 12 & 34 \\
\hline Survival & Survive & Survive & Survive & Survive \\
\hline
\end{tabular}

Abbreviations: DM, diabetes mellitus; HTN, hypertension; SND, selective neck dissection. 
A

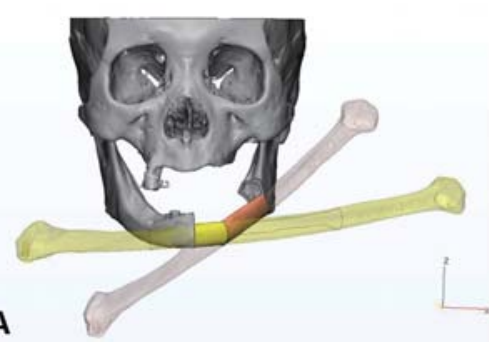

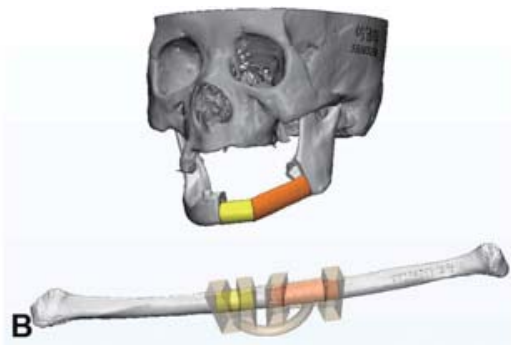



Fig. 1 Preoperative computer simulation and three-dimensional (3-D) printing cutting guide. The patient had an oromandibular defect on her left side after cancer resection. The defect involved the tongue base; thus, we planned to perform a dynamic oromandibular reconstruction. Once the plan to use a fibular free flap was established, we used 3-D computer simulation to manufacture a 3-D printing cutting guide (Jeil Medical, Seoul, Korea). (A, B) Preoperative simulation. (C) 3-D printing cutting guide.
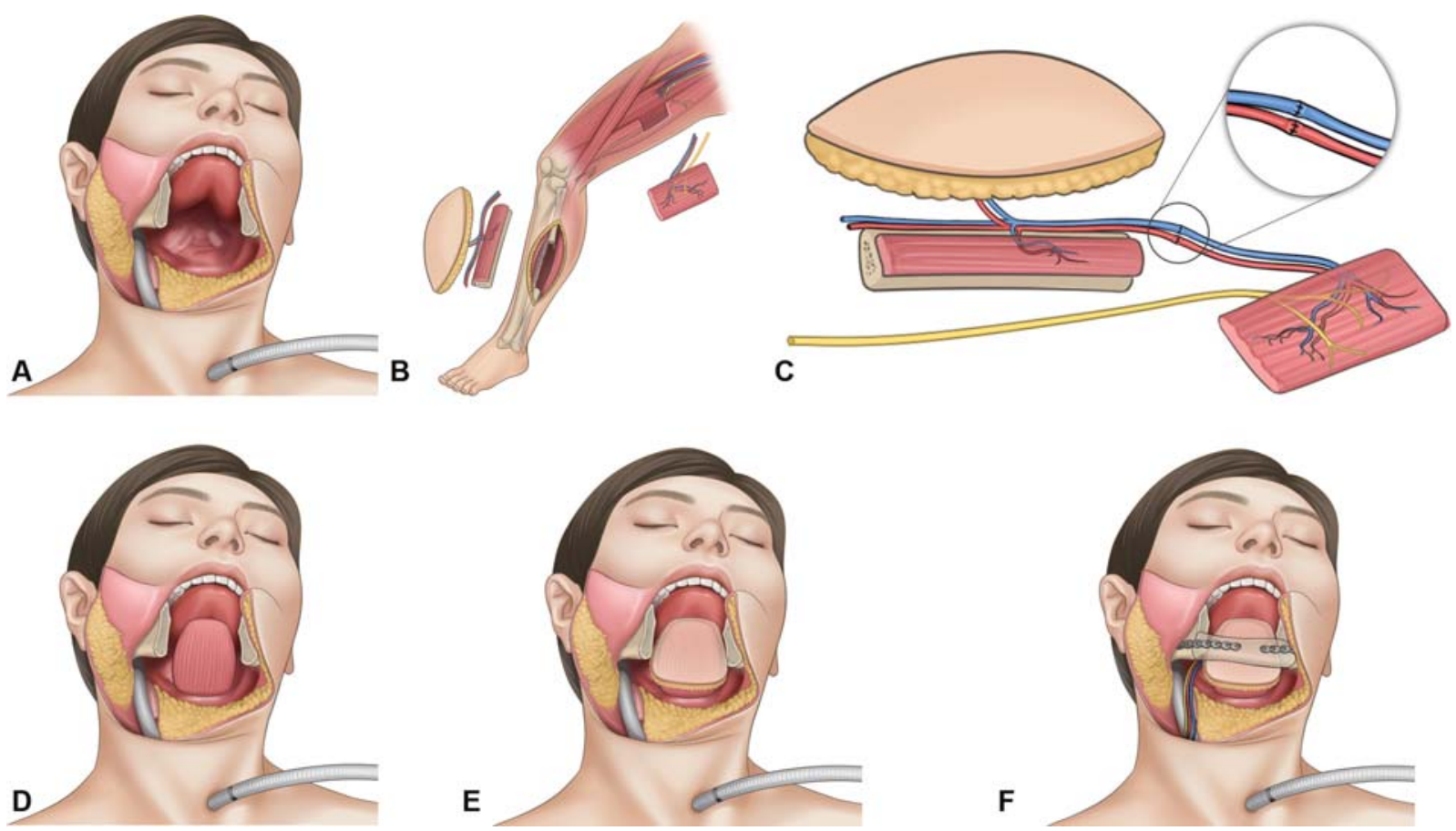

Fig. 2 Schematic illustration of the concept of dynamic oromandibular reconstructions. (A) A 51-year-old woman who had an extensive oromandibular defect that involved the oral floor and base of the tongue underwent segmental mandibulectomy involving teeth 31-33 and 41-44. (B) We decided to reconstruct the chimeric fibular free flap with functional gracilis transfer. (C) The distal pedicle of the fibular flap was anastomosed to the pedicle of the gracilis muscle. Neurorrhaphy was performed between the obturator nerve of the gracilis muscle and the remaining hypoglossal nerve. The following illustrations depict the insertion of the chimeric flap step by step. (D) The gracilis flap was inserted first. Its origin was sutured with the tongue defect, and the insertion was connected to the genioglossus mandibular attachment. (E) The fibular skin flap was overlaid on the gracilis muscle, which resulted in water tightness. (F) Finally, the fibular bone flap can occupy the mandibular defect.

dissection with resection of the segmental mandibulectomy involving teeth 31 to 33 and 41 to 44 . The right inferior alveolar nerve was cut, and the genioglossus, hyoglossus, and geniohyoid muscles were resected to secure a safe margin. The mandibular defect was reconstructed with a free fibular flap, and two segmental osteotomies were performed to achieve the mandibular shape. A $4 \times 2 \mathrm{~cm}$ of gracilis muscle flap was harvested with the obturator nerve. Its pedicle medial circumflex artery and vein were anastomosed with the distal pedicle of the fibular free flap. After the completion of the distal anastomosis, the fibular free flap was fully mobilized. A $7 \times 4 \mathrm{~cm}$ size skin paddle of fibular flap covered the oral floor, and the gracilis muscle lay beyond the skin flap. The gracilis muscle flap was inserted in the direction of the genioglossus for mimicking its function. Eight miniscrews were used for intermaxillary fixation. The facial artery and vein were used as recipient vessels anastomosed end-to-end to the peroneal artery and vein. Both flaps survived well, and complete wound healing was attained uneventfully (-Table 2; -Fig. 3).

\section{Case 2: Chimeric Anterolateral Free Flap with the Vastus Lateralis}

A 52-year-old woman with a recurrent gingival squamous cell carcinoma underwent tumor resection with skin invasion involving the mentum and right submandible. A right midline segmental mandibulectomy was performed, and three-fourths of the tongue was resected with the oral floor and extrinsic muscle above the hyoid bone level. The left upper gingiva was resected including the anterior pillar and right tonsil. The main mass invasion visibly extended to the right medial pterygoid 
Table 2 Operative details of the study population

\begin{tabular}{|l|l|l|l|l|}
\hline Patient no. & $\mathbf{1}$ & $\mathbf{2}$ & $\mathbf{3}$ & $\mathbf{4}$ \\
\hline Type of reconstruction & Dynamic & Dynamic & Control & Control \\
\hline Mandibular defect size $(\mathrm{cm})$ & $8 \times 4$ & $7 \times 4$ & $5 \times 3$ & $6 \times 5$ \\
\hline Type of flap & Fibular free flap & Fibular free flap & Fibular free flap & Fibular free flap \\
\hline Conjoined flap & Gracilis & Vastus lateralis & $(-)$ & $(-)$ \\
\hline Pedicle length $(\mathrm{cm})$ & 8 & 9 & 8 & 7 \\
\hline Flap dimension $(\mathrm{cm})$ & & & & \\
\hline Skin & $7 \times 4$ & $15 \times 8$ & $5 \times 3$ & $7 \times 5$ \\
\hline Muscle & $4 \times 2$ & $8 \times 5$ & $(-)$ & $(-)$ \\
\hline Bone & 6 & 8 & 4 & 6 \\
\hline Microanastomosis & & & & \\
\hline Number of A. & 1 & 1 & 1 & 1 \\
\hline Number of V. & 1 & 2 & 2 & 1 \\
\hline Recipient A. & Facial A. & Facial A. & STA & STA \\
\hline Recipient V. & Facial V. & Facial V. & IJV, EJV & Facial V. \\
\hline Recipient N. & Hypoglossal N. & Hypoglossal N. & $(-)$ & $(-)$ \\
\hline Number of bone segments & 2 & 3 & 2 & 2 \\
\hline Donor site coverage & STSG & STSG & STSG & STSG \\
\hline Flap survival & Survive & Survive & Survive & Survive \\
\hline Complications & $(-)$ & Wound dehiscence & $(-)$ & $(-)$ \\
\hline
\end{tabular}

Abbreviations: A, artery; EJV, external jugular vein; IJV, internal jugular vein; N, nerve; STA, superior thyroidal artery; STSG, split-thickness skin graft; V, vein.
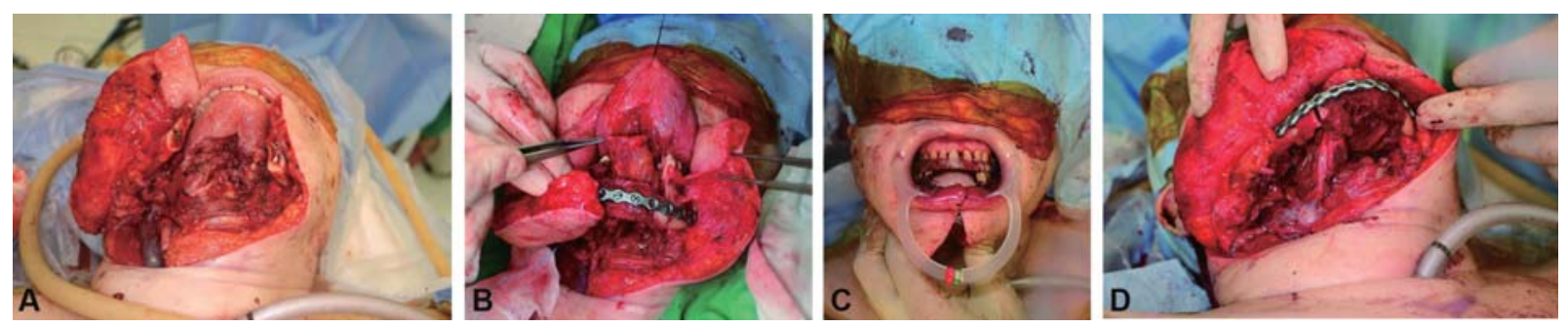

Fig. 3 Intraoperative photograph of patient 1. (A) Base of the tongue in the tongue-mandible defect. (B) Chimeric flap insertion. (C) Immediate postoperative feature. (D) Pedicle insertion.

and masseter muscles. A vascularized fibular osteocutaneous flap was designed for the anterior and right mandibular body. A simultaneously harvested anterolateral thigh free flap was designed to cover the right external skin defect, and the tongue defect was covered with a vastus lateralis muscle flap. An anterolateral thigh skin paddle of $15 \times 8 \mathrm{~cm}$ in size and an $8 \times 5 \mathrm{~cm}$ muscle flap were harvested. The descending branches of the lateral circumflex artery and vein were anastomosed with the distal pedicle of the fibular flap. The peroneal artery and vein were anastomosed to the right facial artery and vein. The motor branch of the femoral nerve that innervated the vastus lateralis was anastomosed end-to-side with the hypoglossal nerve (-Table 2; - Fig. 4).

\section{Neurorrhaphy}

In both cases, the hypoglossal nerve was used as the recipient nerve. The hypoglossal nerve innervates most of the extrinsic and intrinsic muscles of the tongue and is involved in controlling the tongue movements required for speech and swallowing. Therefore, the hypoglossal nerve as the sole motor nerve was selected as the recipient nerve and anastomosed to the obturator nerve, which supplies the gracilis muscle, and branch of the femoral motor nerve, which supplies the vastus lateralis muscle. The ear, nose, and throat (ENT) team preserved the hypoglossal nerve; thus, we anastomosed it end-to-side with Ethilon 8-0.

\section{Functional Assessment}

For the objective assessment of surgical outcomes, we categorized the outcomes into three subspecializations, namely speech, swallowing, and aesthetics. For speech evaluation, a trained speech therapist evaluated the patient outcomes. Tongue ROM upon protrusion, lateralization, and elevation, and the total performance severity score were evaluated. Then, the total tongue ROM score was calculated from (Protrusion + Right Lateralization + Left

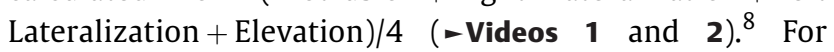



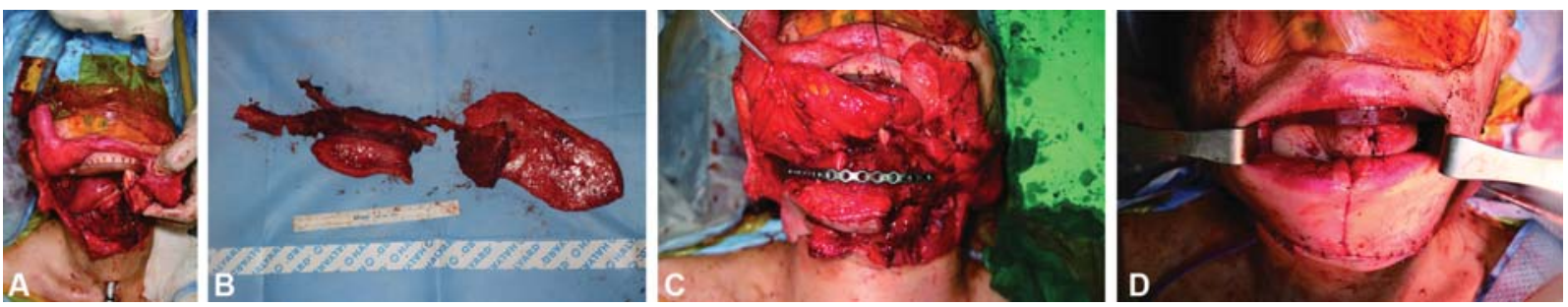

Fig. 4 Intraoperative photograph of patient 2. (A) Defect involving the mentum and right submandibular skin treated with midline segmental mandibulectomy with resection of three-fourths of the tongue. (B) Chimeric flap insertion. (C) Postoperative photograph. (D) Immediate postoperative feature.

proper swallowing evaluation, we used magnetic resonance imaging (MRI), which is a dynamic imaging modality. Multiphase MRI scans were taken after the intravenous injection of a contrast agent. MRI shows characteristics of the time-intensity curve of the regions of interest. Therefore, dynamic MRI has recently gained attention for the subjective and semiquantitative analysis of time-intensity curves. ${ }^{9}$ Our institution recently developed an imaging modality that enables evaluation of patients who have undergone dynamic reconstruction ( - Video 3 ). Lastly, for aesthetic evaluation, in the era of mandibular reconstruction, the final facial contour was evaluated. Postoperative 3-D photographs of all the selected patients were obtained with a Morpheus 3-D scanner (Morpheus Co., Ltd., Seongnam City, Gyeonggi-do, Korea). We compared hemifacial 3D volumes. A similar facial volume was considered to indicate well-reconstructed facial bony contour.

\section{Video 1}

Speech evaluation of patient 1 (dynamic group). Online content including video sequences viewable at: https://www.thieme-connect.com/products/ejournals/html/10.1055/s-0040-1713801.

\section{Video 2}

Speech evaluation of patient 3 (control group). Online content including video sequences viewable at: https://www.thieme-connect.com/products/ ejournals/html/10.1055/s-0040-1713801.

\section{Video 3}

Swallowing evaluation with dynamic magnetic resonance imaging (MRI) of patient 1 (dynamic group). Online content including video sequences viewable at: https://www.thieme-connect.com/products/ ejournals/html/10.1055/s-0040-1713801.

\section{Results}

The four patients included one man and three women, with a mean age of 57 years at the time of reconstruction. Patients 1 and 2 underwent dynamic reconstruction with a chimeric free fibular flap. Patients 3 and 4 underwent conventional reconstruction with a free fibular flap only. Two patients had oral floor cancer, and the other two had gingival cancer. The mean follow-up period was 17.5 months, and all the patients received radiotherapy during the postoperative period, except patient 2 who refused postoperative treatment. All the flaps survived without major complications such as flap rejection ( $\mathbf{- T a b l e} \mathbf{1})$.

In the speech outcome analysis, the dynamic group showed satisfactory tongue movements. Protrusion and lateralization were the movements that showed the most remarkable changes among others in patients who underwent conventional reconstruction. The total tongue ROM score was 62.5 in the dynamic group and 25.0 in the control group. Both groups had similar levels of intelligibility, but the articulation correction was more superior in the dynamic group (-Table 3).

In the 3-D volumetric analysis of mandibular aesthetic contouring, the dynamic group showed a smaller difference in hemifacial volume (73.7 vs. $101.76 \mathrm{~mL}$; - Table 3).

Patient 1 underwent dynamic MRI ( - Table 3 ). The patient was instructed to gulp continuously several times to allow contrast media to flow. According to the tracking MRI, we demonstrated the tongue movement and swallowing action. The contact of the soft palate with the tongue was excellent, and epiglottis closure was performed when glutition was complete. Pharyngeal constrictor muscle contraction was evidently visible.

In the videofluoroscopic swallow test, the dynamic and control groups were evaluated ( - Table 4 ). The pharyngeal and esophageal phases appeared similar in both groups; however, the oral phase in the control group showed more mobilization during chewing motion. The overall function of the dynamic group was not inferior to that of the control group.

\section{Discussion}

Given the fact that the tongue and mandible are the most dynamic structures in our body, we believe that dynamic reconstruction should be performed more while focusing on comprehensive anatomical and functional restoration of oromandibular defects. For mandibular bony defects, the consensus 
Table 3 Objective functional outcome assessment of the study population

\begin{tabular}{|l|l|l|l|}
\hline & Patient no. & $\mathbf{1}$ & $\mathbf{3}$ \\
\hline \multirow{5}{*}{ Speech outcome } & Type of reconstruction & Dynamic & Control \\
\hline \multirow{5}{*}{} & Postoperative period (mo) & 10 & 10 \\
\cline { 2 - 4 } & Protrusion & Mildly impaired & Totally impaired \\
\cline { 2 - 4 } & Right lateralization & Normal & Mildly impaired \\
\cline { 2 - 4 } & Left lateralization & Mildly impaired & Totally impaired \\
\cline { 2 - 4 } & Elevation & Mildly impaired & Mildly impaired \\
\cline { 2 - 4 } & Total tongue ROM score (/100) & 62.5 & 25 \\
\cline { 2 - 4 } & Articulation & 90.69 & 78.2 \\
\cline { 2 - 4 } & Intelligibility & Mild & Mild \\
\hline Three-dimensional volumetric analysis (mL) & Hemifacial right & 848.47 & 998.18 \\
\cline { 2 - 4 } & Hemifacial left & 774.77 & 896.34 \\
\cline { 2 - 4 } & Difference & 73.7 & 101.76 \\
\hline \multirow{5}{*}{ Dynamic MRI } & & $(+)$ & $(-)$ \\
\cline { 2 - 4 } & Soft palate & Contact & \\
\cline { 2 - 4 } & Epiglottis & Contraction & \\
\cline { 2 - 4 } & Pharyngeal constrictor muscle & \\
\hline
\end{tabular}

Abbreviations: MRI, magnetic resonance imaging; ROM, range of motion.

Table 4 Results of the videofluoroscopic swallow test

\begin{tabular}{|c|c|c|c|}
\hline & Patient no. & 2 & 4 \\
\hline & Type of reconstruction & Dynamic & Control \\
\hline \multirow[t]{7}{*}{ Oral phase } & Lip sealing & Enough & Enough \\
\hline & Chewing & Not enough & Not enough \\
\hline & Tongue control & Enough & Enough \\
\hline & Velar elevation & Enough & Enough \\
\hline & Oral transit time & 0.001 & 0.001 \\
\hline & Piecemeal swallow & No & No \\
\hline & Residue in mouth & No & No \\
\hline \multirow[t]{9}{*}{ Pharyngeal phase } & Aspiration amount & No & No \\
\hline & Nasal regurgitation & No & No \\
\hline & Pharyngeal transit time & 0.001 & 0.001 \\
\hline & Amount of food in the vallecular pouch & No & No \\
\hline & Residue in the pyriform sinus & No & No \\
\hline & Coating of the pharynx walls after swallow & No & No \\
\hline & Opening of the pharyngoesophageal segment & Intact & Intact \\
\hline & Triggering of pharyngeal swallow & Normal & Normal \\
\hline & Laryngeal elevation and epiglottic closure & Normal & Normal \\
\hline Esophageal phase & $\begin{array}{l}\text { Mechanical obstruction } \\
\text { Delayed passage } \\
\text { Gastroesophageal reflux }\end{array}$ & $\begin{array}{l}\text { No } \\
\text { No } \\
\text { No }\end{array}$ & $\begin{array}{l}\text { No } \\
\text { No } \\
\text { No }\end{array}$ \\
\hline Functional dysphagia scale & & 3 & 3 \\
\hline ASHA NOMS & & 6 & 7 \\
\hline
\end{tabular}

Abbreviations: ASHA NOMS, American Speech-Language Hearing Association National Outcome Measurement System Swallowing Scale. 
is that the fibular osteocutaneous flap is an excellent option. The fibular free flap provides support for dental implantation and dentures, helping in the recovery of occlusal function. Recently, a patient-specific virtual surgical planning and 3-D printing cutting guide was developed to enable reconstruction with successful aesthetic and functional outcomes. ${ }^{10,11}$ However, with regard to the tongue, only few trials of dynamic tongue reconstruction have been conducted. Thus, we hypothesized that functional muscle transfer would be a solution for the restoration of the hyoid-jaw-tongue complex. Reports have demonstrated a cortical reorganization after motor nerveinnervated microneurovascular muscle transfer. ${ }^{12}$ By adapting these biomechanics, we believe that the hypoglossal motor nerve can provide a powerful and reliable innervation to the transferred muscle. A transferred musculo/musculocutaneous flap can mimic oral soft tissue anatomical structures. The extrinsic and intrinsic muscles of the tongue actively affect tongue movements, and their relationship with the jaw also affects the base of the tongue. The hypoglossal nerve plays a major role in these activities. Therefore, our dynamic oromandibular reconstruction uses a fibular flap for bony coverage and innervated functional muscle and skin flaps for comprehensive coverage of the soft tissues.

The best option for functional muscle transfer in head and neck reconstruction must be identified. First, we searched for the most valuable options. In our cases, we demonstrated that functional muscle transfer is possible even in oral reconstruction. The gracilis and vastus lateralis muscles facilitated successful functional reconstruction. The prerequisites were as follows: First, the muscle should have a reliable motor nerve with sufficient length. The obturator nerve can be easily harvested when the pedicle dissected and the motor nerve branch of the vastus lateralis travel medially alongside the descending branch vessels. Second, the muscle should have a favorable muscle fiber direction. It is evident that reconstruction of all 26 extrinsic and intrinsic muscles is impossible. Therefore, we decided to target muscle function. Among the various extrinsic tongue muscles, we aimed at the genioglossus or styloglossus muscle, which allows protrusion and swallowing through a sling action. Third, in the interest of time, two teams participated in the chimeric flap harvest. For this, the ipsilateral upper thigh was easy to access. While the ENT team resected the cancer lesion, the plastic surgery team harvested the fibular free flap. Then, we proceeded to harvest one more chimeric flap and simultaneously prepared the recipient vessel and nerve. This is a preliminary trial of dynamic oromandibular reconstruction; thus, we planned to identify optimal options for functional muscle transfer. We believe an integrated reconstruction of the jaw-tongue complex and an innervated functional muscle transfer could be an effective approach in similar cases.

\section{Conclusion}

This study is a preliminary trial of dynamic oromandibular reconstruction using a chimeric free fibular flap with functional muscle transfer. Although the number of cases was limited, through this study, we demonstrated the possibility of dynamic oromandibular reconstruction, which enhances more functional aspects in the patients.

Funding

None declared.

Conflict of Interest

None declared.

\section{References}

1 Nakatsuka T, Harii K, Yamada A, Ueda K, Ebihara S. Dual free flap transfer using forearm flap for mandibular reconstruction. Head Neck 1992;14(06):452-458

2 Wei FC, Celik N, Chen HC, Cheng MH, Huang WC. Combined anterolateral thigh flap and vascularized fibula osteoseptocutaneous flap in reconstruction of extensive composite mandibular defects. Plast Reconstr Surg 2002;109(01):45-52

3 Hiiemae KM, Palmer JB. Tongue movements in feeding and speech. Crit Rev Oral Biol Med 2003;14(06):413-429

4 Lass N, McReynolds L, Northern J, Yoder D. Speech, Language, and Hearing, Volume 11, Pathologies of Speech and Language. Philadelphia: WB Saunders Co.; 1982

5 Thexton AJ, McGarrick JD. Tongue movement in the cat during the intake of solid food. Arch Oral Biol 1989;34(04):239-248

6 Hiiemae K, Palmer J. Tongue-jaw linkages: the mechanisms of feeding revisited. Bull Mus Comp Zool 2001;156(01):205-217

7 Chen HH, Qiu SS, Lin CH, Kang CJ, Liao CT. Fibula osteoseptocutaneous flap: advantages of beginning the harvesting from the posterior approach. Ann Plast Surg 2016;77(06):635-639

8 Lazarus CL, Husaini H, Jacobson AS, et al. Development of a new lingual range-of-motion assessment scale: normative data in surgically treated oral cancer patients. Dysphagia 2014;29(04): 489-499

9 Chikui T, Obara M, Simonetti AW, et al. The principal of dynamic contrast enhanced MRI, the method of pharmacokinetic analysis, and its application in the head and neck region. Int J Dent 2012; 2012:480659

10 Chang EI, Jenkins MP, Patel SA, Topham NS. Long-term operative outcomes of preoperative computed tomography-guided virtual surgical planning for osteocutaneous free flap mandible reconstruction. Plast Reconstr Surg 2016;137(02):619-623

11 Toto JM, Chang EI, Agag R, Devarajan K, Patel SA, Topham NS. Improved operative efficiency of free fibula flap mandible reconstruction with patient-specific, computer-guided preoperative planning. Head Neck 2015;37(11):1660-1664

12 Manktelow RT, Tomat LR, Zuker RM, Chang M. Smile reconstruction in adults with free muscle transfer innervated by the masseter motor nerve: effectiveness and cerebral adaptation. Plast Reconstr Surg 2006;118(04):885-899 